Pensamiento Crítico Vol. 18 № 1, pp. 81-104

\title{
Economía de Las Universidades \\ Nacionales (1990-2010)
}

Juan José Manuel Ysaac Ospino Edery

\section{RESUMEN}

Esta investigación analiza la legislación universitaria para el periodo 1970-2010, el presupuesto fijado a las universidades estatales, discriminando aquel designado a las ubicadas en la ciudad de Lima, como aquel destinado a las de provincias, que a fines de nuestro periodo de análisis llegan a un numero de 46.

Apreciándose que el estado, viene sustrayendo su apoyo a los presupuestos de la universidad estatal, las que han priorizado el gasto corriente frente a los de capital, la implementación de la ley del Canon minero y las universidades públicas receptoras y sus limitaciones en el gasto, así como un análisis comparativo de las asignaciones del presupuesto con el sistema de universidades de países del grupo andino (GRAN).

Palabras claves: Presupuesto público. Asignación presupuestal. Gasto Corriente. Gasto de capital. Economía de las Universidades. 


\title{
Pensamiento Crítico Vol. 18. $\mathrm{N}^{\circ}$ I
}

\begin{abstract}
This research for university law review for period 1970-2010, the analysis fixed budget to state universities, discriminating that designed those locates in the city of Lima, and intended for provincial.

If follows that the state is supported by subtracting the budgets of state university, Which have prioritized spending compared to current capital, the implementation of Canon law mining and public universities receiving and the amounts received in the last five years, and limitations on spending. As final point, a comparative analysis of budget allocations to the university system of Andean group of countries.
\end{abstract}

\section{INTRODUCCIÓN}

La economía de las universidades nacionales nos hace referencia a las asignaciones presupuestales que reciben del estado, al Canon, Regalías y a los ingresos propios que generan a las donaciones que recogen de organizaciones privadas o estatales, pudiendo ser estas nacionales o extranjeras, así como su distribución para gastos, los cuales están clasificados en dos rubros, gastos corrientes (remuneraciones, bienes, transferencias corrientes y pensiones) y gastos de capital.

Presupuestos que se inician de manera deficitaria cuando se consideran las asignaciones que hace cada año el gobierno en los pliegos correspondientes, situación que ha llevado a las universidades estatales a desarrollar mecanismos ingeniosos para generarse ingresos y cubrir sus requerimientos económicos, que en algunos casos representa un monto cercano al $50 \%$ del total que se destina a gastos, como se aprecia en el cuadro siguiente Nro.1

Cabe resaltar que en concordancia con la Ley General de Educación, la educación superior es la segunda etapa del sistema educativo que consolida la formación integral de las personas, produce conocimiento, desarrolla la investigación e innovación y forma profesionales en el más alto nivel de especialización y perfeccionamiento en todos los campos del saber, el arte, la cultura, la ciencia y la tecnología. 


\section{Juan José Manuel Ysaac Ospino Edery}

Cuadro N.1. Presupuesto de Apertura por Fuente de Financiamiento Universidades Estatales

\section{Millones de nuevos soles corrientes}

\begin{tabular}{ccccccc}
\hline Año & \multicolumn{2}{c}{ Presupuesto Total } & \multicolumn{2}{c}{ Tesoro Publico } & \multicolumn{2}{c}{ Ingresos propios } \\
\hline 1995 & 583 & $100 \%$ & 495 & $85 \%$ & 87 & $15 \%$ \\
2000 & 1045 & $100 \%$ & 736 & $70 \%$ & 308 & $30 \%$ \\
2005 & 1511 & $100 \%$ & 1007 & $67 \%$ & 504 & $33 \%$ \\
2010 & 2346 & $100 \%$ & 1455 & $62 \%$ & 891 & $38 \%$ \\
\hline
\end{tabular}

Fuente: Asamblea Nacional de Rectores. Resumen Estadístico Universitario 2010. Elaboración propia.

La universidad no ha sido ajena a los vaivenes de nuestra economía y sociedad, la crítica situación económica y política presentada en la década de los 80 y con mayor incidencia durante el periodo de gobierno 1985 al 90 que llevaron al país a niveles de inflación sin precedentes, presento una coyuntura que resto avances significativos tanto en lo educativo como en su infraestructura.

Las medidas de ajuste estructural de carácter económico llevadas a cabo por el gobierno del presidente Fujimori, si bien lograron remontar la hiperinflación, estas fueron acompañadas de una serie de políticas de carácter neoliberal, orientadas a contraer el apoyo del estado al sistema universitario estatal, condiciones que se hacen manifiestas en nuestra constitución vigente.

De manera puntual se tiene que nuestra actual constitución señala con respecto a la educación lo siguiente:

"Artículo 14.- La educación promueve el conocimiento, el aprendizaje y la práctica de las humanidades, la ciencia, la técnica, las artes, la educación física y el deporte. Prepara para la vida y el trabajo y fomenta la solidaridad.

Es deber del Estado promover el desarrollo científico y tecnológico del país, La formación ética y cívica y la enseñanza de la Constitución y de los derechos humanos son obligatorias en todo el proceso educativo civil o militar. La educación religiosa se imparte con respeto a la libertad de las conciencias. 


\section{Pensamiento Crítico Vol. I8. N I}

La enseñanza se imparte, en todos sus niveles, con sujeción a los principios constitucionales y a los fines de la correspondiente institución educativa.

Los medios de comunicación social deben colaborar con el Estado en la educación y en la formación moral y cultural".

Como se aprecia en el renglón precedente, no se indica que en las asignaciones presupuestales para los gastos en educación se tomen como referencia a porcentajes del PBI, situación contradictoria cuando casi todas las economías modernas consideran los gastos sociales como porcentajes de este importante indicador macroeconómico, aspecto que si estuvo reconocido en nuestra constitución del año 1933.

\section{La legislación Universitaria 1970-2010}

La junta militar (Junta de Gobierno Revolucionario) que se instituyo en octubre del 68, dado su marco ideológico de carácter nacionalista estatista, planteo una nueva reforma universitaria a la cual denomino "Ley Orgánica de la Universidad Peruana", o D.L. No 17437 del 18 de febrero de 1969, medida que introdujo innovaciones substanciales en el que hacer universitario como:

- La conformación de los Departamentos y Programas académicos que sustituyeron a las facultades, la creación del régimen semestral, los estudios generales y el currículum flexible así como la del Consejo Nacional de la Universidad Peruana (CONUP), máximo organismo de dirección y representación del sistema de la Universidad.

- En Marzo de 1972, se promulgo la Ley Orgánica de Educación No 19326 "Reforma Educativa”, derogándose el D.L. No 17437, la cual convoca una comisión Estatutaria para que elabore el Estatuto de la Universidad Peruana, que no fue aprobado por el gobierno, originándose un vacío legal, llevando a la universidad a una situación de crisis de administración y autoridad.

- El año 1977 mediante DL 21925 promulgado por el presidente Francisco Morales Bermúdez, se renovaron todas las autoridades del sistema universitario, con personas identificadas al gobierno, circunstancia que agravo la crisis de dirección de las universidades. 


\section{Juan José Manuel Ysaac Ospino Edery}

- El año 1980 se reinstauro la democracia, en el cual es reelegido el presidente Fernando Belaúnde Terry, el cual promulgo en 1983 la Ley No 23733 "Ley Universitaria", la que cancelo el impopular sistema de departamentos y programas restaurando la organización académica de facultades.

- El 20 de Enero de 1995, la Ley Nro. 26439 para la creación del Consejo Nacional para la Autorización de funcionamiento de Universidades (CONAFU).

- El 8 de Noviembre de 1996 el DL 882 a la cual se le denomino ley de Promoción de la Inversión en Educación (universidad empresa), promulgados por el presidente Alberto Fujimori. Dispositivos más trascendentes entre otros que se añaden a la ley Nro. 23733, y que a la fecha norman a nuestro sistema universitario.

Tanto los objetivos como el espíritu de estos decretos fueron, el dar cauce legal a una mayor participación del sector privado en la educación, para lo cual se modifica la normativa vigente para la creación de universidades, así como limitar la participación del estado en lo que respecta a medidas económicas de carácter presupuestal, comprometiendo a las universidades estatales a desarrollar una serie de actividades que lindan con un contexto de privatización para la generación de recursos económicos, con los cuales puedan operar.

Es de resaltar que a partir de los años cincuenta, la aparición de nuevas universidades tanto en la capital de la república, como en los principales departamentos al interior, así como el incremento de las migraciones principalmente de la zona de la sierra central hacia la costa, trajeron consigo un cambio en la estructura de los postulantes y futuros universitarios, estos ya en su mayoría provendrían de todas las sangres, de un país mestizo como el nuestro.

Una conclusión temprana resultante de los acontecimientos vistos hasta el momento, nos indica que no ha habido una continuidad de la normativa y del espíritu de la legislación universitaria en los últimos cincuenta años, aspectos que han contribuido a un funcionamiento anárquico de nuestro sistema universitario, el mismo que se encuentra desarticulado de una educación secundaria pobre e insuficiente. 


\section{Pensamiento Crítico Vol. I8. Nº}

\section{Presupuesto asignado a las Universidades Estatales.}

Las universidades estatales despliegan su acción académica principalmente con un presupuesto determinado por el estado y de manera adicional en el caso de algunas Universidades con los recursos que generan y otras con el aporte adicional del Canon y Regalías mineras, financian una buena proporción sus gastos.

De acuerdo a la ley Nro. 28322, a los gobiernos regionales les corresponde el $25 \%$ del total del canon minero (50\% del impuesto a la renta). Las cuales se obligan a transferir el $20 \%$ de esa suma a las universidades públicas de su jurisdicción, de acuerdo a la ley Nro. 28077 de Setiembre del $2003^{1}$. Por otro lado reciben un 5\% por regalías minera.

El detalle de la evolución de las asignaciones del tesoro público al presupuesto de las universidades estatales, así como las fuentes de ingresos puede ser apreciado en el cuadro Nro. 2 que abarca el periodo que va de 1994 al 2010.

En esta etapa se registro un crecimiento sostenido en las aportaciones del tesoro público cercano a 265\% entre los años 1994 al 2010, mientras que el requerimiento de presupuesto total representa un $397 \%$ de incremento en similar periodo. La diferencia registrada entre lo total presupuestado y asignado vía fuente del tesoro viene siendo cubierto con la fuente de ingresos propios (generados por la universidad) el cual de un aporte de 90 millones para el año 94, registra un aumento de 891 millones los que equivale a un incremento en el monto cercano al 847\% para el año 2010.

Podemos interpretar que el estado viene limitando, sustrayendo su apoyo a los presupuestos de la universidad estatal, lo cual obedecería a la implementación de políticas económicas que se orientan a racionalizar, restringir su rol en diferentes actividades y en que la actividad educativa se encuentra incluida. La distribución de los gastos, tanto aquellos de carácter corriente y de capital, en que los primeros tienen como principal

1 A las universidades públicas les toca el $5 \%$ del total del canon transferido, (20\% del 25\%) solo para aquellas situadas en jurisdicciones donde se realicen actividades mineras $\mathrm{u}$ otras que generen canon y regalías. Este deberá ser destinado de manera exclusiva a la inversión en investigación científica y tecnológica. 


\section{Juan José Manuel Ysaac Ospino Edery}

componente los sueldos de los docentes y no docentes y a los que se suman el pago de pensiones registra un crecimiento de sustantivo de más del 500\% entre 1994 al 2010².

Tenemos que subrayar que en lo que respecta a los gastos de capital los mismos consideran renovación y compra de equipamiento, obras de infraestructura para la universidad, ha presentado un crecimiento menor al corriente lo que explica el deterioro sustantivo de la infraestructura de la universidad nacional aspecto que se hace más patente en aquellas que se encuentran fuera de la ciudad capital.

Cuadro N.ํ 2. Evolución Del Presupuesto* de La Universidad Estatal (1994-2010)

\section{(En millones de nuevos soles corrientes)}

\begin{tabular}{|c|c|c|c|c|c|c|c|c|c|c|c|c|}
\hline $\mathbf{a}$ & b & & & d & & & & & $\mathbf{g}$ & & & $\mathbf{i}$ \\
\hline \multirow{2}{*}{ Año } & \multirow{2}{*}{ Total } & \multirow{2}{*}{$\begin{array}{l}\text { Tesoro } \\
\text { Pubico }\end{array}$} & \multirow{2}{*}{$\begin{array}{c}\text { Otros } \\
\text { (1) }\end{array}$} & \multirow{2}{*}{$\begin{array}{c}\text { Ingresos } \\
\text { Propios } \\
\mathrm{s} /\end{array}$} & \multicolumn{2}{|c|}{$\begin{array}{l}\text { Gasto } \\
\text { Corriente }\end{array}$} & \multicolumn{2}{|c|}{$\begin{array}{l}\text { Gasto de } \\
\text { Capital }\end{array}$} & \multirow[t]{2}{*}{$\begin{array}{l}\text { Nro. } \\
\text { Alumno }\end{array}$} & \multicolumn{2}{|c|}{$\begin{array}{c}\text { Percapita } \\
\text { b/g }\end{array}$} & \multirow[t]{2}{*}{$\begin{array}{c}\text { Nro. } \\
\text { Universid }\end{array}$} \\
\hline & & & & & s/ & $e / b \%$ & $\mathrm{~s} /$ & $\mathrm{f} / \mathrm{b} \%$ & & s/ & US\$ & \\
\hline 1994 & 472 & 382 & 12 & 90 & 370 & 78.4 & 102 & 21.6 & 242133 & 1949 & 890 & 28 \\
\hline 2000 & 1045 & 725 & 12 & 308 & 914 & 87.5 & 131 & 12.5 & 254732 & 4102 & 1175 & 32 \\
\hline 2005 & 1511 & 1007 & 18 & 486 & 1298 & 85.6 & 212 & 14.4 & 281374 & 5370 & 1627 & 35 \\
\hline $2010 e$ & 2348 & 1395 & 60 & 891 & 1876 & 80.0 & 470 & 12.0 & 309175 & 7587 & 2681 & 40 \\
\hline 2009 & 2129 & 1308 & 55 & 766 & 1852 & 86.9 & 271 & 13.1 & 300404 & 7087 & 2354 & 38 \\
\hline $2010 e$ & 2346 & 1395 & 60 & 891 & 1876 & 80.0 & 470 & 12.0 & 309175 & 7587 & 2681 & 40 \\
\hline
\end{tabular}

Fuente: Presupuesto institucional de apertura. Resumen Estadístico Universitario año 2007. Dirección de De Planificación Universitaria. Asamblea Nacional de Rectores. Memorias Banco Central de Reserva 1990/2006. Elaboración propia. *Apertura. Estimaciones y Proyecciones de población 1950- 2050 INEI. Ministerio de Economía y Finanzas 2010.

Ingresos por Canon y Sobrecanon. e. Estimado

\section{Presupuesto asignado a las Universidades Estatales de la Ciudad de Lima}

Apreciando el cuadro Nro.3, este nos indica que durante el periodo que va del año 2000 al 2010, las universidades ubicadas tanto en la ciudad de Lima como en

2 Este incremento en el Gasto podría atribuirse a los pagos por la homologación de los sueldos de los Docentes Universitarios la que se cumple en un $100 \%$ a partir del año 2010. 


\section{Pensamiento Crítico Vol. I8. Nº}

las provincias presentaron un incremento nominal de sus presupuestos lo que podría atribuirse, al aumento del número de universidades así como al incremento de los haberes de los docentes universitarios por la homologación realizada a partir del año 2010.

\section{Cuadro $\mathbf{N} .^{\circ}$ 3. DISTRIBUCIÓN GEOGRAFICA DEL PRESUPUESTO DE LAS UNIVERSIDADES PÚBLICAS LIMA - PROVINCIAS}

(Soles corrientes)

\begin{tabular}{ccccccccc}
\hline Años & Lima* S/ & \% & Universi & $\begin{array}{c}\text { Gasto } \mathbf{x} \\
\text { Alumno }\end{array}$ & Provincias S/ & $\%$ & UNiversi & $\begin{array}{c}\text { Gasto x } \\
\text { Alumno }\end{array}$ \\
\hline 2000 & 478074181 & 42 & 5 & 3224 & 668317000 & 58 & 27 & 2592 \\
2005 & 570744054 & 39 & 5 & 6668 & 907054000 & 61 & 30 & 4756 \\
2006 & 621358482 & 39 & 5 & 6657 & 944161000 & 61 & 30 & 4915 \\
2007 & 697697026 & 39 & 5 & 6600 & 1102973000 & 61 & 31 & 4900 \\
$2010 e$ & $772103010 e$ & 33 & 6 & 12373 & 1573896990 & 67 & 34 & 6378 \\
\hline
\end{tabular}

Fuente: Resumen Estadístico Universitario 2000-2010. Asamblea Nacional de Rectores. Elaboración propia

* No se considera a las Universidades José Faustino Sánchez Carrión de Huacho- Universidad Nacional del Callao

Consideramos también que las asignaciones per cápita por alumno son mayores en las universidades de Lima respecto a las de provincia, situación que se atribuye a los mayores costos que demanda la operación de la Universidades de la capital.

Los cuadros siguientes Nro.4 al Nro.8, nos permitirán un análisis particular del comportamiento de los presupuestos de las Universidades Nacionales más representativas ubicadas en la ciudad de Lima, así tenemos que la evolución del presupuesto de la UNMSM que se aprecia en el cuadro Nro.4, para el periodo que va de 1995 al 2010 ha presentado una tendencia progresiva lo cual nos explica una capacidad adquisitiva creciente tomando como base el año 2001, en este periodo de tiempo la universidad ha venido financiando parte de su presupuesto con recursos propios directamente recaudados en montos que llegan cerca del $48 \%$ del total presupuestado. 


\section{Juan José Manuel Ysaac Ospino Edery}

Cabe indicar que la población estudiantil registró un crecimiento del $17.8 \%$ entre los periodos 1995 al 2010, proporción menor al incremento de las asignaciones presupuestales per cápita para el periodo antes mencionado la misma que fue del orden del $120 \%$, situación que ha conllevado a mejoras sustantivas en su infraestructura, recursos físicos, tecnológicos y bibliográficos. ${ }^{3}$

Cuadro N. ${ }^{\circ}$ 4. PRESUPUESTO DE LA UNMSM en (soles) Capacidad Adquisitiva y Per cápita año base 2001

\begin{tabular}{ccccccc}
\hline Años & $\begin{array}{c}\text { Presupuesto } \\
\text { Nuevos Soles }\end{array}$ & $\begin{array}{c}\text { Presupuesto } \\
\text { Crecimiento } \\
\text { Periodo \% }\end{array}$ & $\begin{array}{c}\text { Capacidad } \\
\text { Adquisitiva }\end{array}$ & $\begin{array}{c}\text { Alumnos } \\
\text { Matricula }\end{array}$ & $\begin{array}{c}\text { Alumnos } \\
\text { Crecimiento } \\
\text { Periodo \% }\end{array}$ & $\begin{array}{c}\text { Per } \\
\text { cápita } \\
\text { S/ }\end{array}$ \\
\hline 1995 & 117160502 & ------ & 65.20 & 24305 & ------ & 4820 \\
2000 & 202378137 & 72.7 & 112.60 & 25363 & 6.37 & 7979 \\
2001 & 179716261 & -11.2 & 100.00 & 27479 & 8.34 & 6540 \\
2005 & 243500933 & 10.17 & 135.49 & 29144 & -0.16 & 8355 \\
2010 & 305190013 & 11.3 & 169.81 & 28645 & 0.39 & 10654 \\
\hline
\end{tabular}

Fuente: Universidad Nacional Mayor de San Marcos. Compendios estadísticos 1995-2011. Elaboración propia.

Durante el segundo lustro que va del 2000 al 2010 la Oficina General de Infraestructura universitaria, muestra que en facultades se construyeron alrededor de 44,000 mts cuadrados. Cabe señalar que a nivel internacional la recomendación es de 1.50 mts Cuadrados por cada alumno como mínimo, en la UNMSM el indicador es de 1.34 mts Cuadrados ${ }^{4}$.

En el cuadro siguiente Nro.5 se aprecia la evolución del presupuesto de la Universidad Nacional de Ingeniería en el periodo que va de 1995 al 2010 el cual tuvo un incremento del $208 \%$ financiado en buena parte con recursos propios los cuales en los últimos años del periodo representan montos superiores al $50 \%$ de sus requerimientos.

3 El compendio estadístico 2011, en el punto de información financiera señala, que para el año 2010 el $54 \%$ del presupuesto corresponde a recursos ordinarios asignados por el estado y $45 \%$ corresponde a recursos directamente recaudados por la universidad.

En lo referente a la ejecución presupuestal se realizo en un 94\%, (287 millones) donde el programa de Educación superior demando un gasto de 53.5\% mientras que el programa de gestión un $30.7 \%$.

4 Comentario del documento Compendio Estadístico 2011 de la UNMSM, sección Infraestructura, Recursos Tecnológicos. 


\section{Pensamiento Crítico Vol. I8. Nº}

"En 1989 la administración de la UNI, propuso a la comunidad universitaria que, a la luz del análisis de la situación que entonces vivía la sociedad peruana poco podía esperarse del Gobierno Central y del Congreso de la Republica para proveer de fondos que el quehacer universitario requiere. El planteamiento ante esta situación: una política de generación de recursos propios activando, mediante un reglamento promotor, a todos los miembros de la comunidad y órganos de la institución para que se avocaran a producirla.

El objetivo principal formulado entonces y vigente hasta hoy fue el de desarrollar una base económica propia que posibilite la ejecución de los planes, actividades y proyectos con importante participación de los recursos directamente generados por la UNI y una cada vez menor dependencia de las fuentes de recursos ordinarios" ${ }^{5}$.

Cuadro $\mathbf{N} .^{\circ}$ 5. Presupuesto de La Universidad Nacional de Ingenieria

Capacidad Adquisitiva y Per cápita año base 2001 (soles)

\begin{tabular}{ccccccc}
\hline Años & $\begin{array}{c}\text { Presupuesto } \\
\text { Nuevos } \\
\text { Soles }\end{array}$ & $\begin{array}{c}\text { Presupuesto } \\
\text { Crecimiento } \\
\text { Periodo \% }\end{array}$ & $\begin{array}{c}\text { Capacidad } \\
\text { Adquisitiva }\end{array}$ & $\begin{array}{c}\text { Alumnos } \\
\text { Matricula }\end{array}$ & $\begin{array}{c}\text { Alumnos } \\
\text { Crecimiento } \\
\text { Periodo \% }\end{array}$ & $\begin{array}{c}\text { Per } \\
\text { cápita }\end{array}$ \\
\hline 1995 & 62897000 & --------- & 55.96 & 7861 & ------- & 8000 \\
2000 & 100121235 & 12.00 & 89.07 & 10476 & -3.51 & 9557 \\
2001 & 112394741 & 12.25 & 100.00 & 10345 & -1.25 & 10864 \\
2005 & 126235071 & 4.76 & 112.31 & 10558 & 4.57 & 11956 \\
$2010 e$ & 193825709 & 22.8 & 193.59 & 11034 & 0.45 & 17566 \\
\hline
\end{tabular}

Fuente: Memoria institucional UNI 2010.

Cabe resaltar que su población estudiantil para el periodo 1995-2010 registro un crecimiento del $40 \%$, porcentaje menor al incremento de las asignaciones presupuestales per cápita para el periodo que fue del orden del $119 \%$, este incremento sustantivo en los ingresos generados por la Universidad ha traído como resultado un mejoramiento de la infraestructura académica que la coloca en un lugar expectante dentro de las Universidades estatales.

5 Memoria Universidad Nacional de Ingeniería 1998. Capitulo 9 ejecución presupuestal. 


\section{Juan José Manuel Ysaac Ospino Edery}

Para el último año de nuestro análisis la UNI registra ingresos propios cercanos al $66 \%$, situación que se aprecia en la grafica Nro.1, resultante de las activas actividades que realizan sus centros de producción.

Grafico N. ${ }^{\circ}$ 1. UNI Ejecución de Presupuesto-Ingresos 2010

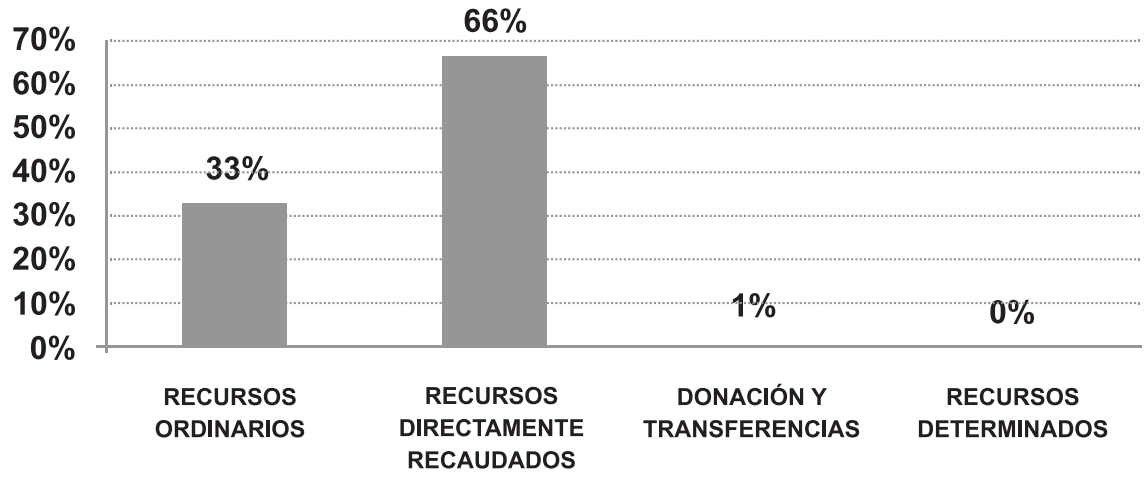

Fuente: Memoria institucional UNI 2010.

En el cuadro Nro.6 se aprecia que el presupuesto de la Universidad Nacional Agraria La Molina ha presentado un incremento del $78.7 \%$ en el periodo que va de los años 1995 al 2010, aumento que ha venido siendo financiado en los cinco últimos años del periodo con alrededor de un $20 \%$ de recursos directamente recaudados y la diferencia con recursos ordinarios, cabe resaltar que en el periodo de análisis la población estudiantil se vio incrementada en un $35 \%$, mientras que el presupuesto per cápita en un $32 \%$ lo que nos estaría indicando que a pesar de los esfuerzos que vienen realizando la Universidad para generar fondos se estaría dando un deterioro en su estructura de gastos por alumno.

Cuadro N. ${ }^{\circ}$ 6. Presupuesto de La Universidad Nacional Agraria La Molina Capacidad Adquisitiva y Per cápita año base 2001(soles)

\begin{tabular}{ccccccc}
\hline Años & $\begin{array}{c}\text { Presupuesto } \\
\text { Nuevos Soles }\end{array}$ & $\begin{array}{c}\text { Presupuesto } \\
\text { Crecimiento } \\
\text { Periodo \% }\end{array}$ & $\begin{array}{c}\text { Capacidad } \\
\text { Adquisitiva }\end{array}$ & $\begin{array}{c}\text { Alumnos } \\
\text { Matricula }\end{array}$ & $\begin{array}{c}\text { Alumnos } \\
\text { Crecimiento } \\
\text { Periodo \% }\end{array}$ & Per cápita \\
\hline 1995 & 37100000 & ------- & 90.48 & 3629 & -------- & 10223 \\
2000 & 38000000 & 5.30 & 92.68 & 4943 & 16.15 & 7687 \\
2001 & 41000000 & 7.89 & 100.00 & 5116 & 3.50 & 8014
\end{tabular}




\section{Pensamiento Crítico Vol. I8. N I}

\begin{tabular}{ccccccc}
2005 & 52000000 & 3.46 & 126.82 & 5871 & 3.05 & 8857 \\
$2010 e$ & 66303600 & 14.67 & 161.71 & 4903 & 2.14 & 13523 \\
\hline
\end{tabular}

Fuente: Universidad Nacional Agraria. Compendios estadísticos 1995-2007. Elaboración propia. e. estimado

En el cuadro Nro.7 se aprecia la evolución del presupuesto de la Universidad Federico Villareal para el periodo 1995-2010 espacio de tiempo en el cual se registra un incremento del $72 \%$ el cual ha venido siendo financiado en los últimos cinco años con alrededor del $40 \%$ con recursos directamente recaudados y la diferencia con recursos ordinarios del tesoro.

Cuadro N. ${ }^{\circ}$ 7. Presupuesto de La Universidad Nacional Federico Villarreal

Capacidad Adquisitiva y Per cápita año base 2001

\begin{tabular}{ccccccc}
\hline Años & $\begin{array}{c}\text { Presupuesto } \\
\text { Nuevos Soles }\end{array}$ & $\begin{array}{c}\text { Presupuesto } \\
\text { Crecimiento } \\
\text { Anual \% }\end{array}$ & $\begin{array}{c}\text { Capacidad } \\
\text { Adquisitiva }\end{array}$ & $\begin{array}{c}\text { Nro. de } \\
\text { Alumnos }\end{array}$ & $\begin{array}{c}\text { Alumnos } \\
\text { Crecimiento } \\
\text { Anual \% }\end{array}$ & $\begin{array}{c}\text { Per } \\
\text { cápita }\end{array}$ \\
\hline 1995 & 90000000 & ------------ & 83.33 & 21856 & --------- & 4117 \\
2000 & 104000000 & 2.97 & 96.27 & 17688 & -9.36 & 5879 \\
2001 & 108000000 & 3.84 & 100.00 & 20759 & 16.35 & 5202 \\
2005 & 121687985 & 4.09 & 112.67 & 25127 & 5.18 & 4842 \\
2010 & 154898892 & 22.2 & 148.9 & 26900 & 2.3 & 5758 \\
\hline
\end{tabular}

Fuente: Universidad Nacional Federico Villareal. Compendios estadísticos 1994-2010. Elaboración propia.

En lo que respecta a la evolución de la población de alumnado entre el inicio y fin del periodo analizado presenta un crecimiento del orden del $23 \%$ mientras que la distribución per cápita del presupuesto ha presentado un incremento cercano a un $39 \%$, lo que nos estaría indicando una mejora en la asignación por alumno.

En el cuadro siguiente Nro.8 se considera la evolución del presupuesto de la Universidad Enrique Guzmán y Valle (UNE), para el periodo 1995-2010 periodo de tiempo en el cual se registra un incremento del $115 \%$ el cual ha venido siendo financiado en los últimos cinco años con alrededor del $30 \%$ con recursos directamente recaudados y la diferencia un $70 \%$ con recursos ordinarios asignados por el tesoro, distribuidos en alrededor de un $78 \%$ como gasto corriente (personal y obligaciones previsionales y 


\section{Juan José Manuel Ysaac Ospino Edery}

otros gastos corrientes) y un $22 \%$ como gastos de capital (inversión en equipamiento), prorrateo que nos indica el por qué del deterioro la obsolescencia e insuficiente de la infraestructura física de la Universidad.

Cuadro $\mathbf{N}^{\circ}$.8. PRESUPUESTO DE LA UNIVERSIDAD ENRIQUE GUZMAN Y VALLE

Capacidad Adquisitiva y Per cápita año base 2001

\begin{tabular}{ccccccc}
\hline Años & $\begin{array}{c}\text { Presupuesto } \\
\text { Nuevos Soles }\end{array}$ & $\begin{array}{c}\text { Tasa de } \\
\text { Crecimiento } \\
\text { Anual \% }\end{array}$ & $\begin{array}{c}\text { Capacidad } \\
\text { Adquisitiva }\end{array}$ & $\begin{array}{c}\text { Población } \\
\text { Nro. de } \\
\text { Alumnos }\end{array}$ & $\begin{array}{c}\text { Tasa de } \\
\text { Crecimiento } \\
\text { Anual \% }\end{array}$ & $\begin{array}{c}\text { Per } \\
\text { cápita }\end{array}$ \\
\hline 1995 & 29000000 & ------------- & 83.59 & 15165 & ------------ & --------- \\
2000 & 32571000 & 5.26 & 93.89 & 5615 & -1.17 & 5800 \\
2001 & 34690000 & 6.50 & 100.00 & 5548 & -1.19 & 6253 \\
2005 & 44875992 & 6.14 & 129.36 & 8746 & -7.10 & 5131 \\
2010 & 44875992 & 6.14 & 129.36 & 8746 & -7.10 & 5131 \\
\hline
\end{tabular}

Fuente: Universidad Nacional Enrique Guzmán y Valle. Compendios estadísticos 1994-2010. Elaboración propia. e: Estimado

En lo que respecta a la evolución de la población de alumnado entre el inicio y fin del periodo analizado presenta un decremento del orden del 39\% mientras que la distribución per cápita del presupuesto ha presentado un incremento cercano a un $17 \%$, lo que nos estaría mostrando una relativa mejora de las asignaciones por alumno.

Hay que resaltar que la UNE, es considerada como la más representativa formadora de profesionales en educación en el Perú y que al igual que la mayoría de las universidades nacionales tanto por la finalidad de generarse ingresos para financiar su presupuesto, como para incentivar una mayor demanda por los servicios educativos que ofrece, implementa a partir de Agosto del 2003 su centro preuniversitario CEPREUNE, a la cual se le asigna un $30 \%$ anual de las vacantes a ofertar para un ingreso directo.

\section{Presupuesto asignado a las Universidades Estatales de Provincias}

El cuadro precedente Nro.9 nos indica la existencia de 46 universidades en provincias, siendo las décadas de los años 1960, 2000 y 2010 donde se constituyen 9 , 


\section{Pensamiento Crítico Vol. I8. Nº}

9 y 13 respectivamente (alrededor del 69\% del total), cabe destacar que la fundación de las mismas en la mayoría de los casos obedeció al interés político de sus gestores y sin mayor sustento de su planificación académica ${ }^{6}$.

Son las universidades San Antonio Abad del Cuzco, Universidad de Trujillo, San Agustín, San Luis Gonzaga y Pedro Ruiz Gallo las que demandan más de un 30\% de los presupuestos iníciales de apertura asignado a las 46 universidades de provincia, monto que registra un incremento destacable entre el periodo 2003 al 2011 de alrededor de un $100 \%{ }^{7}$

La distribución de los gastos corrientes (personal obligaciones sociales, pensiones, prestaciones sociales, bienes y servicios, donaciones y transferencias) del último periodo (2011) en promedio es de un $83 \%$, la diferencia $17 \%$ para gastos de capital, monto que nos señala la pobre asignación que se realiza a lo que son inversiones en infraestructura, compra de nuevo equipamiento y reposición, cabe resaltar que las universidades San Agustín, y la del Santa asignan alrededor del 95\% de sus presupuestos para gastos corrientes, seguidas de las universidades Daniel Alcides Carrión y la Jorge Basadre con más del $90 \%$.

Una de las tres funciones de la universidad es realizar investigación, la que es muy pobre dada las limitadas asignaciones económicas para este fin, situación que es más grave en la universidades de provincias a la que se agrega también las retribuciones para proyección social, cumpliendo de manera limitada lo que es la docencia, vale decir el desarrollo de un mayor capital humano.

6 La ley $\mathrm{N}^{\circ} 29971$ (Diciembre 21 del 2012), establece la moratoria de 5 años para la creación y autorización de funcionamiento de nuevas universidades públicas y privadas, y la suspensión de creación de filiales en el interior del país por el mismo período. La finalidad de la moratoria es permitir el replanteo de la política de la educación superior universitaria dentro de una nueva legislación, en el marco de un sistema de educación que establezca requisitos de creación y funcionamiento de universidades debidamente acreditadas y certificadas, que garanticen calidad, investigación.

La ley dispone "excepcionalmente", que el Consejo Nacional para la Autorización de Funcionamiento de Universidades (CONAFU) evalúe y autorice durante el plazo de un año el funcionamiento de las universidades públicas que hayan superado la fase de verificación de la implementación inicial. Las demás universidades creadas por ley continúan su correspondiente trámite.

7 Apreciación obtenida del Resumen Estadístico Universitario 2010. Dirección General de Planificación Universitaria publicado el 2012. Asamblea Nacional de rectores. 


\section{Juan José Manuel Ysaac Ospino Edery}

Apreciando la fuente de financiamiento que se registra para el periodo 2011, a diferencia de las universidades nacionales de la capital, que mediante recursos directamente recaudados financian alrededor de un $45 \%$ de sus gastos, porcentaje que viene incrementándose de manera anual, las universidades de provincia lo hacen con alrededor de un 23\%, siendo las universidades San Agustín, Pedro Ruiz Gallo y La Santiago Antúnez de Mayolo las que lo sufragan con alrededor de un $30 \% .{ }^{8}$ Se puede atribuir estas menores captaciones a una menor actividad de sus centros de producción, dado el pequeño mercado en la que se desenvuelven.

Cuadro N. ${ }^{\circ}$ 9. Universidades Nacionales de Provincias por año de creación Canon Recibido

\begin{tabular}{|c|c|c|c|c|c|c|c|}
\hline & Universidad & Sede & Creación & $\begin{array}{l}\text { Canon } \\
\text { Minero }\end{array}$ & $\begin{array}{c}\text { PIA } \\
2000\end{array}$ & $\begin{array}{c}\text { PIA } \\
2003\end{array}$ & $\begin{array}{c}\text { PIA } \\
2011\end{array}$ \\
\hline 1 & U,N de San Antonio Abad & Cusco & 01-06-1692 & $\mathrm{x}$ & $53,334,000$ & $58,267,000$ & $113,345,032$ \\
\hline 2 & U.N de Trujillo & Trujillo & $10-05.1824$ & $\mathrm{x}$ & $53,165,000$ & $58,096,000$ & $112,469,292$ \\
\hline 3 & U.N de San Agustín & Arequipa & 02-06-1827 & $\mathrm{x}$ & $75,500,000$ & $80,096,000$ & $149,599,565$ \\
\hline 4 & U.N San Luis Gonzaga & Ica & 20-12-1955 & $\mathrm{x}$ & $45,850,000$ & $50,063,000$ & $104,927,236$ \\
\hline 5 & U.N San Cristóbal de Huamanga & Ayacucho & 24-04-1957 & $\mathrm{x}$ & $30,750,000$ & $35,805,189$ & $64,520,715$ \\
\hline 6 & U.N del Centro del Perú & Huancayo & $16-12-1959$ & $\mathrm{x}$ & $32,850,000$ & $39,017,161$ & $73,972,459$ \\
\hline 7 & U.N de la Amazonia & Iquitos & 14-01-1961 & $\mathrm{x}$ & $35,378,000$ & $41,741,000$ & $62,450,993$ \\
\hline 8 & U.N del Altiplano & Puno & $10-02-1961$ & $\mathrm{x}$ & $45,546,000$ & $50,043,848$ & $92,582,547$ \\
\hline 9 & U.N de Piura & Piura & 03-03-1961 & $\mathrm{x}$ & $37,712,000$ & $42,785,000$ & $97,830,617$ \\
\hline 10 & U.N de Cajamarca & Cajamarca & 13-02-1962 & $\mathrm{x}$ & $28,591,000$ & $33,308,000$ & $60,961,338$ \\
\hline 11 & U.N Hermilio Valdizan & Huánuco & $22-02-1964$ & $\mathrm{x}$ & $18,509,000$ & $23,270,000$ & $44,520,796$ \\
\hline 12 & U.N Agraria de la Selva & Tingo María & 20-02-1964 & $\mathrm{x}$ & $12,661,000$ & $17,154,359$ & $31,782,126$ \\
\hline 13 & U.N Daniel Alcides Carrion & $\begin{array}{l}\text { Cerro de } \\
\text { Pasco }\end{array}$ & 23-04-1965 & $\mathrm{x}$ & $19,554,000$ & $22,148,000$ & $47,223,910$ \\
\hline 14 & U.N del Callao & Callao & 02-09-1966 & & $25,281,000$ & $36,154,000$ & $67,790,663$ \\
\hline 15 & U.N José F. Sánchez Carrión & Huacho & $31-12-1968$ & $\mathrm{x}$ & $19,718,000$ & $28,976,000$ & $55,689,403$ \\
\hline 16 & U.N Pedro Ruiz Gallo & Lambayeque & $17-03-1970$ & $\mathrm{x}$ & $43,661,000$ & $50,454,000$ & $110,490,733$ \\
\hline 17 & U.N Jorge Basadre Grohmann & Tacna & 01-08-1971 & $\mathrm{x}$ & $20,532,000$ & $25,463,607$ & $44,435,616$ \\
\hline 18 & U.N Santiago Antúnez de Mayolo & Huaraz & 24-05-1977 & $\mathrm{x}$ & $15,681,000$ & $21,313,868$ & $43,216,939$ \\
\hline 19 & U.N de San Martin & Tarapoto & 18-12-1979 & $\mathrm{x}$ & $9,525,000$ & $13,532,000$ & $31,496,511$ \\
\hline 20 & U.N de Ucayali & Pucallpa & $18-12-1979$ & & $9,017,000$ & $12,252,000$ & $30,027,305$ \\
\hline
\end{tabular}

8 Ibíd. 7. 


\section{Pensamiento Crítico Vol. 18. Nº}

\begin{tabular}{|c|c|c|c|c|c|c|c|}
\hline 21 & U.N de Tumbes & Tumbes & 23-06-1984 & & $8,406,000$ & $12,540,000$ & $27,386,699$ \\
\hline 22 & U.N del Santa & Chimbote & $20-12-1984$ & $\mathrm{x}$ & $6,406,000$ & $11,383,142$ & $17,945,557$ \\
\hline 23 & U.N de Huancavelica & Huancavelica & 20-06-1990 & $\mathrm{x}$ & $8,290,000$ & $11,379,826$ & $27,657,504$ \\
\hline 24 & U.N Intercultural de la Amazonia & Ucayali & $30-12-1999$ & & $3,100,000$ & $3,920,000$ & $9,800,644$ \\
\hline 25 & $\begin{array}{l}\text { U.N Amazónica de Madre de } \\
\text { Dios }\end{array}$ & $\begin{array}{c}\text { Puerto } \\
\text { Maldonado }\end{array}$ & $05-07-2000$ & $\mathrm{x}$ & $4,950,000$ & $5,290,000$ & $12,901,099$ \\
\hline 26 & $\begin{array}{l}\text { U.N Toribio Rodríguez de } \\
\text { Mendoza }\end{array}$ & Chachapoyas & $18-09-2000$ & $\mathrm{x}$ & $4,350,000$ & ND & $22,339,990$ \\
\hline 27 & $\begin{array}{l}\text { U.N Micaela Bastidas de } \\
\text { Apurímac }\end{array}$ & Abancay & $22-09-2000$ & $\mathrm{x}$ & ND & ND & $13,320,353$ \\
\hline 28 & U.N Tecnológica del Cono Sur de & Lima & $01-02-2001$ & & ND & ND & $15,997,909$ \\
\hline 29 & U.N José María Arguedas & Andahuaylas & $29-10-2004$ & $\mathrm{x}$ & ND & ND & $12,193,770$ \\
\hline 30 & U.N de Moquegua & Moquegua & $12-05-2005$ & & ND & ND & $13,959,796$ \\
\hline 31 & U.N de Juliaca & Juliaca & $20-07-2007$ & & & & \\
\hline 32 & U.N de Jaén & Jaen & $19-12-2008$ & & & & \\
\hline 33 & U.N de Cañete (2) & Cañete & $22-12-2009$ & & & & \\
\hline 34 & U.N Autónoma de Chota (1) & Chota & $11-05-2010$ & & & & \\
\hline 35 & U.N de Barranca (1) & Barranca & 06-07-2010 & & & & \\
\hline 36 & U. N de Frontera (1) & Sullana & 05-08-2010 & & & & \\
\hline 37 & $\begin{array}{l}\text { U.N Intercultural "Fabiola Salazar } \\
\text { Leguía" (2) }\end{array}$ & Bagua & $17-11-2010$ & & & & \\
\hline 38 & $\begin{array}{l}\text { U.N Intercultural de la Selva } \\
\text { Central Juan Santos Atahualpa (3) }\end{array}$ & Satipo & $18-11-2010$ & & & & \\
\hline 39 & U.N Intercultural de Quillabamba (3) & Quillabamba & $30-11-2010$ & & & & \\
\hline 40 & $\begin{array}{l}\text { U.N Autónoma de Alto } \\
\text { Amazonas (4) }\end{array}$ & Yurimaguas & $10-01-2011$ & & & & \\
\hline 41 & U.N Altoandina de Tarma (2) & Tarma & $13-01.2011$ & & & & \\
\hline 42 & U.N Autónoma de Huanta (2) & Huanta & $27-01-2011$ & & & & \\
\hline 43 & $\begin{array}{l}\text { U.N Tecnológica de San Juan de } \\
\text { Lurigancho (3) }\end{array}$ & $\begin{array}{l}\text { San Juan de } \\
\text { Lurigancho }\end{array}$ & $27-01-2011$ & & & & \\
\hline 44 & $\begin{array}{l}\text { U. Autónoma Municipal de los } \\
\text { Olivos (4) }\end{array}$ & Los OLivos & 09-03-2011 & & & & \\
\hline 45 & $\begin{array}{l}\text { U. Autónoma de Tayacaja Daniel } \\
\text { Hernández Morillo (3) }\end{array}$ & Pampas & $22-06-2011$ & & & & \\
\hline 46 & U.N Ciro Alegría (3) & Huamachuco & $16-07-2011$ & & & & \\
\hline
\end{tabular}

Fuente: ANR. Estadísticas Universitarias.2000- 2012. 1) En proceso de implementación. 2) Admitidos a trámite. 3) Solo cuenta con ley de creación. 4) Solo cuenta con ley de creación, Funciona con recursos que provienen del Gobierno Municipal.PIA (Presupuesto inicial de apertura- en nuevos soles). 


\section{Juan José Manuel Ysaac Ospino Edery}

\section{Universidades Públicas y Canon Minero}

Determinadas universidades reciben montos adicionales para financiar sus presupuestos los mismos que provienen del Canon Minero el cual es el más representativo, otros Canon, sobre Canon y regalías.

"Según el ordenamiento jurídico peruano esta es la participación efectiva y adecuada que gozan los Gobiernos Locales (municipalidades provinciales y distritales) y los Gobiernos Regionales del total de ingresos y rentas obtenidas por el Estado por la explotación económica de los recursos mineros (metálicos y no metálicos).

Así, efectuada la recaudación del impuesto a la renta, el estado por intermedio de la Secretaría de Descentralización de la Presidencia del Consejo de Ministros (PCM) transfiere un porcentaje de ingresos captados por dicho concepto a las zonas en donde se explotó el recurso minero"9.

La distribución del canon ( $50 \%$ del impuesto a la renta que pagan las empresas mineras), se distribuye de la siguiente manera:

- El $10 \%$ del total recaudado para los gobiernos de la municipalidad o municipalidades distritales donde se encuentra localizado el recurso natural.

- El 25\% del total recaudado para los gobiernos locales de la provincia donde se encuentra localizado el recurso natural, excluyendo al distrito o distritos productores.

- El $40 \%$ del total recaudado para los gobiernos locales del departamento o departamentos de las regiones excluyendo a la provincia donde se encuentra el recurso natural.

- El 25\% del total recaudado para los gobiernos regionales donde se encuentra el recurso natural ,de este porcentaje, el $20 \%$ será entregado a las universidades públicas de su circunscripción, destinado exclusivamente a la inversión en investigación científica y tecnológica que potencien el desarrollo regional,

9 Tomado del Boletín de la Sociedad Nacional de Minería, Petróleo y Energía. 2010 


\section{Pensamiento Crítico Vol. I8. Nº}

entendiéndose como el gasto en obras de infraestructura y equipamiento. Los porcentajes de asignación serán los mismos para cada universidad de una región, vale decir que se entrega en partes iguales para cada una.

Las investigaciones deberán estar relacionadas con la salud pública y prevención de enfermedades endémicas, sanidad agropecuaria, preservación de la biodiversidad y el ecosistema de la zona geográfica de influencia, donde se desarrollan las actividades económicas extractivas y utilización eficiente de energías renovables y de procesos productivos $^{10}$.

Si bien la normativa del canon para las universidades busca desarrollar y fortalecer las capacidades de investigación, esta ha tenido una menor incidencia, incentivando por lo general el uso del canon en infraestructura y equipamiento y no en actividades de investigación.

Aquí una interrogante, ¿Por qué no se invirtió el canon en mayores investigaciones?, intentaremos algunas respuestas. No hay una visión del rol de algunas universidades de su compromiso con la investigación, hay una dispersión de los esfuerzos al interior de las mismas relacionada con la investigación, o los docentes no saben investigar o nunca han investigado.

El uso del porcentaje del canon minero asignado a las universidades restringe la utilización de este al pago de remuneraciones o retribuciones de cualquier índole, aspecto que desincentiva a la docencia universitaria, a lo que se añade a que este no puede destinarse a la constitución de un mayor capital humano por parte de los docentes de las universidades beneficiarias.

El año 2011 se dispuso que las universidades podrían emplear/ asignar hasta el $20 \%$ del canon minero para financiar sus procesos de acreditación (los trabajos, estudios, equipamiento) para alcanzar la acreditación de la institución, o por especialidad tal como lo señala la ley 28740 del SINEACE ${ }^{11}$.

10 Ley Nro. 29289, del Presupuesto Público para el año Fiscal 2009.

11 Así lo informo el Director de la Comisión Nacional de Evaluación y Acreditación Universitaria (CONEAU). Dr. Fernando Delgado de Flor. En reunión con directivos de la UNAS de Arequipa. 


\section{Juan José Manuel Ysaac Ospino Edery}

Se tiene que preponderar, "Solo reciben canon minero las universidades nacionales o publicas ubicadas en los departamentos en donde hay actividad minera, siempre y cuando las minas de su zona hayan generado utilidades en el periodo anterior" ${ }^{\prime 2}$.

Las universidades de manera adicional reciben el 5\%, por concepto de regalías mineras, los cuales se distribuyen en partes iguales en caso exista más de una en una región ${ }^{13}$.

El cuadro siguiente Nro.10 nos presenta la evolución de las asignaciones por canon minero a ser proporcionada a las universidades ubicadas dentro de las regiones, las cuales reciben mayores montos, como consecuencia lógica de aquellas donde la actividad minera es mayoritaria, tal así que tanto la universidad Nacional del Santa y la Santiago Antúnez de Mayolo ubicadas en la región Ancash son las que han recibido los mayores aportes alrededor de 284 millones de soles, seguidas de la Jorge Basadre Grohman de Tacna, San Agustín de Arequipa, de Cajamarca, por 133, 111 y 109 millones respectivamente para el periodo 2006 al 2011.

En Fórum organizado en Noviembre del 2012 por CEDEP y por el proyecto Pro Participación, sobre Canon y la Universidades Públicas de Ancash en la ciudad de Huaraz, concluyo que la gestión de los fondos obtenidos debe considerar: Exigir la transferencia efectiva de parte del gobierno regional del Canon Minero, mayor participación del alumnado y docentes en la planificación de uso de los fondos y priorización de proyectos y actividades, mayor transparencia en el uso de los recursos, mejor organización para incidir en la modificación de normas que limitan el gasto universitario en investigación ${ }^{14}$.

12 Tomado del boletín CAD Nro. 14 de Setiembre del 2005.

13 Un aspecto que limita el gasto se atribuye al momento en que se hacen las transferencias las mismas que se realizan generalmente el mes de Julio de cada año.

14 Las conclusiones a que se arribaron, se considero pueden ser generalizadas a todas la universidades que reciben una proporción del Canon Minero. Economista Dante Cruz Quiñones Coordinador del Regional del Proyecto Pro-Participación Ancash. 


\section{Pensamiento Crítico Vol. 18. $\mathrm{N}^{\circ}$ I}

Cuadro N. ${ }^{\circ}$ 10. Transferencias de Canon Minero a Universidades Nacionales de Regiones 15 Miles de Nuevos soles

\begin{tabular}{|c|c|c|c|c|c|c|}
\hline Región & 2006 & 2007 & 2008 & 2009 & 2010 & 2011 \\
\hline Amazonas & 0.25 & 0.40 & 0.65 & 3.7 & 5.5 & 6.2 \\
\hline $\begin{array}{l}\text { Ancash } \\
\text { - Nacional del Santa } \\
\text { - Nacional Santiago Antúnez de Mayolo }\end{array}$ & 17436.55 & 81417.5 & 65975.4 & 42773.8 & 39112.1 & 37802.3 \\
\hline Apurimac & 418 & 1153.5 & 1127.2 & 600.2 & 37.2 & 100.1 \\
\hline $\begin{array}{l}\text { Arequipa } \\
\text { - Nacional San Agustín }\end{array}$ & 3562.05 & 7876.5 & 22876.3 & 26542.3 & 17375.6 & 33132.4 \\
\hline Ayacucho & 79.35 & 1048.1 & 2060.3 & 475.1 & 1716.2 & 2872.6 \\
\hline $\begin{array}{l}\text { Cajamarca } \\
\text { - Nacional de Cajamarca }\end{array}$ & 17771.6 & 29280.6 & 9167.4 & 11405.2 & 20584.5 & 20883.6 \\
\hline Callao & 0 & 0 & 0 & 0 & 0 & 0 \\
\hline $\begin{array}{l}\text { Cusco } \\
\text { - San Antonio Abad }\end{array}$ & 3361.8 & 13644.2 & 12120.3 & 6763.7 & 5181.9 & 8504.1 \\
\hline Huancavelica & 293.1 & 1895.9 & 2404.7 & 842.7 & 290.6 & 426.8 \\
\hline Huánuco & 45.4 & 523.5 & 386.4 & 134.15 & 82.5 & 216.15 \\
\hline $\begin{array}{l}\text { Ica } \\
\text { - San Luis Gonzaga }\end{array}$ & 1352.7 & 3318.7 & 3432.6 & 5524 & 3367.1 & 10099.4 \\
\hline $\begin{array}{l}\text { Junin } \\
\text { - Nacional del Centro }\end{array}$ & 515 & 5535.4 & 6161.5 & 1945.4 & 3150.15 & 3933.2 \\
\hline $\begin{array}{l}\text { La Libertad } \\
\text { - Nacional de Trujillo }\end{array}$ & 2696.5 & 14169.9 & 13239.95 & 18602.7 & 21116.3 & 22967.05 \\
\hline Lambayeque & 0 & 0.15 & 0 & 13.7 & 5.8 & 25.1 \\
\hline Lima Régimen Especial & 335.2 & 573.9 & 641.6 & 540.9 & 334.1 & 420.2 \\
\hline Lima & 1594.1 & 9387.5 & 8526.7 & 2873.0 & 3290.25 & 4861.25 \\
\hline Loreto & 0 & 0 & 0 & 0 & 0 & 0 \\
\hline Madre de Dios & 1.6 & 2.15 & 2.4 & 2.2 & 2.8 & 6 \\
\hline $\begin{array}{l}\text { Moquegua } \\
\text { - Nacional de Moquegua }\end{array}$ & 13529.6 & 24360.8 & 10571.7 & 19278.2 & 12274.9 & 19625.3 \\
\hline $\begin{array}{l}\text { Pasco } \\
\text { - Nacional Alcides Carrión }\end{array}$ & 2410.9 & 17774.3 & 18859.9 & 5629.1 & 7491.6 & 9085.2 \\
\hline Piura & 0.75 & 0.35 & 0.5 & 1.7 & 1 & 6.4 \\
\hline
\end{tabular}

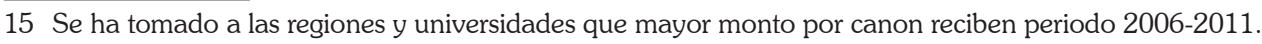




\section{Juan José Manuel Ysaac Ospino Edery}

\begin{tabular}{|c|c|c|c|c|c|c|}
\hline $\begin{array}{l}\text { Puno } \\
\text { - Nacional del Altiplano }\end{array}$ & 5874.7 & 7215.7 & 8625.1 & 12382.7 & 9079.2 & 15358.5 \\
\hline San Martin & 12.8 & 8.2 & 23.9 & 25.6 & 21.8 & 31.1 \\
\hline $\begin{array}{l}\text { Tacna } \\
\text { - Nacional Jorge Basadre Grohman }\end{array}$ & 16026.5 & 38662.5 & 35579.8 & 15362.3 & 9960.3 & 17505.1 \\
\hline Tumbes & 0 & 0 & 0 & 0 & 0 & 0 \\
\hline Ucayali & 0 & 0 & 0 & 0 & 0 & 0 \\
\hline Total & 87318.7 & 257850.05 & 221784.00 & 171722.65 & 154481.8 & 207868.45 \\
\hline
\end{tabular}

Fuente: Ministerio de Economía y Finanzas. DGET. Las universidades de una región reciben el mismo monto por canon. Elaboración propia 2011 a partir del Canon Minero.

\section{Perfil de la economía de Universidades de Países del Grupo Andino "GRAN”.}

Entre los países del GRAN el sistema educativo universitario es considerado solo como mediano, dado que los países integrantes solo cuentan entre un millón y medio millón de estudiantes, para Perú, Chile y Colombia, y pequeño sistema el de Bolivia y Ecuador con menos de medio millón de estudiantes, siendo él mecanismo de ingreso en la mayoría de ellas la de los exámenes de admisión.

Los diversos gobiernos de turno en los últimos treinta años para no ir más allá en la historia han descuidado de manera importante un mayor apoyo presupuestario, tenemos así que el gasto en este sub sector es uno de los más bajos en nuestro continente si lo comparamos con respecto al producto bruto interno y al presupuesto público per cápita para la educación superior en tal como se aprecia en la grafica siguiente, publicadas por la UNESCO ${ }^{16}$.

Valuando la grafica Nro.2, tenemos que el Perú en el periodo 1990-2010 a destinado solo un $0.44 \%$ del PBI como gasto para la educación Universitaria por debajo del $0.60 \%$ de Colombia, del $0.63 \%$ de Chile y del $1.50 \%$ de Bolivia, aquí podemos afirmar la poca importancia que se ha venido dando por el lado de los diferentes gobiernos de turno a la conformación de un capital humano, tan importante para nuestro desarrollo como país.

16 Datos más actualizados de la UNESCO, al año 2005 nos señala la proporción del gasto público en educación como porcentaje del PBI: Bolivia 6.6, Chile 3.8, Colombia 5.0 y Perú 2.6. 


\section{Pensamiento Crítico Vol. 18. Nº}

Es destacable la asignación que realizan tanto Cuba como Bolivia, que a pesar de tener un PBI mucho menor asignan mayores porcentajes a la educación, en el caso del primero este se encuentra cercano a lo cero "0" analfabetismo.

Grafico N. ${ }^{\circ}$ 2. Presupuesto Público para la educación superior universitaria en América Latina como porcentaje del PBI Periodo 1990-2010

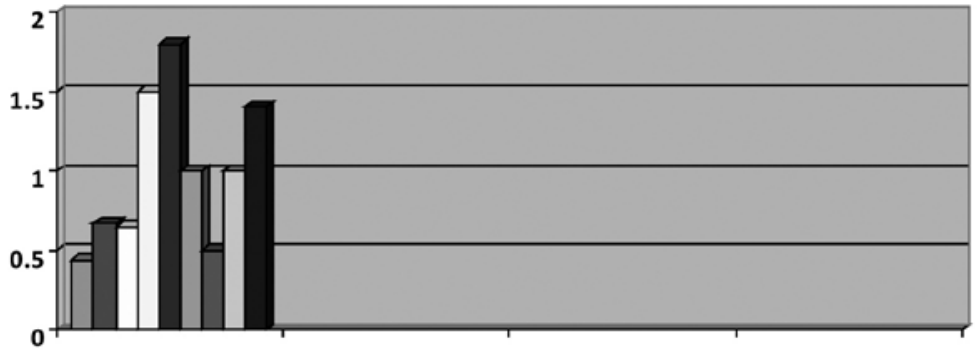

\begin{tabular}{|l|}
\hline$\square$ Perú \\
$\square$ Colombia \\
$\square$ Chile \\
$\square$ Bolivia \\
$\square$ Cuba \\
$\square$ Brasil \\
$\square$ Argentina \\
$\square$ Costa Rica \\
$\square$ Panama
\end{tabular}

Fuente: Tendencias del financiamiento de la educación superior universitaria en America Latina y el Caribe. IELSAC/UNESCO 2010.

Un aspecto que viene haciéndose común y de comportamiento creciente en los países integrantes del GRAN, y que forma parte de su política económica, es que las diferentes universidades estatales financien sus requerimientos presupuestales con los recursos propios que recaben.

\section{Conclusiones}

- No ha habido una continuidad de la normativa y del espíritu de la legislación universitaria en los últimos cincuenta años, escenarios que han contribuido a un funcionamiento anárquico de nuestro sistema universitario.

- Entre los años 1994-2010 se registro un crecimiento sostenido en las aportaciones de la fuente del tesoro público cercano a $265 \%$, mientras que el requerimiento de presupuesto total representa un $397 \%$ de incremento en similar periodo, diferencia cubierta con la fuente de ingresos propios (generados por la universidad. 


\section{Juan José Manuel Ysaac Ospino Edery}

- Si bien la normativa del canon para las universidades busca desarrollar y fortalecer las capacidades de investigación, esta ha tenido una menor incidencia, incentivando por lo general el uso del canon en infraestructura y equipamiento a lo que se agrega que este limita su asignación para mejorar el capital humano, vale decir una mayor capacitación de sus docentes.

- Los diversos gobiernos de turno en los últimos treinta años para no ir más allá en la historia han descuidado de manera importante un mayor apoyo presupuestario, tenemos así que el gasto en este sub sector es uno de los más bajos en nuestro continente.

- La actual y vigente constitución, presenta un vacio dado que no indica que en las asignaciones presupuestales para los gastos de educación, se tome como referencia a porcentaje del $\mathrm{PBI}$, situación contradictoria cuando casi todas las economías modernas consideran los gastos sociales como porcentajes de este importante indicador.

\section{Bibliografía}

ASAMBLEA NACIONAL DE RECTORES. Resumen Estadístico Universitario 19902010. Dirección General de Planificación Universitaria. Lima Perú 2012.

BANCO CENTRAL DE RESERV DEL PERÚ. Indicadores Sociales 1990-2010. Departamentos de Estudios Sector Social. Lima.

DIAZ Juan José. Educación Superior en el Perú. GRDE (Grupo de Análisis para el Desarrollo. Lima 2008.

GRUPO DE ANALISIS PARA EL DESARROLLO (GRADE). Educación Superior en el Perú. Documento de Trabajo Nro.9. Lima1990.

INSTITUTO DE ESTADISTICA E INFORMATICA DEL PERÚ (INEI). Estimaciones y Proyecciones de Población 1950-2050. 


\section{Pensamiento Crítico Vol. I8. N I}

INSTITUTO DE ESTADISTICA E INFORMATICA DEL PERÚ (INEI). Compendio Estadístico 2011.

CUANTO. PERÚ EN NUMEROS 2010. Instituto Cuanto. 2011 Lima.

MINISTERIO DE EDUCACIÓN. La Universidad En el Perú. Dirección de Coordinación Universitaria. Cuadernos de Reflexión y Debate Nro. VI Lima 2006.

UNESCO. INFORME SOBRE EDUCACIÓN SUPERIOR EN EL PERU. 2003.

LEY UNIVERSITARIA 23733. Capitulo XII del Régimen Económico. Artículos del 76 al 89.

GONZALES DE LA CUBA Raúl. El Financiamiento de la Educación Superior en El Perú. Lima 2004.

OSPINO EDERY Ysaac. Estudio de la Concentración de la Población en la Zonas Urbano Marginales en la Ciudad de Lima y la Demanda por Educación Universitaria Estatal 1980-2010. Instituto de Investigaciones Económicas FCE 2011 .UNMSM.

OSPINO EDERYYsaac. La Demanda de Educación Universitaria Estatal una Apreciación 1980-2006. Instituto de Investigaciones Económicas. Revista "Pensamiento Crítico Nro. 8. FCE 2008. UNMSM. 